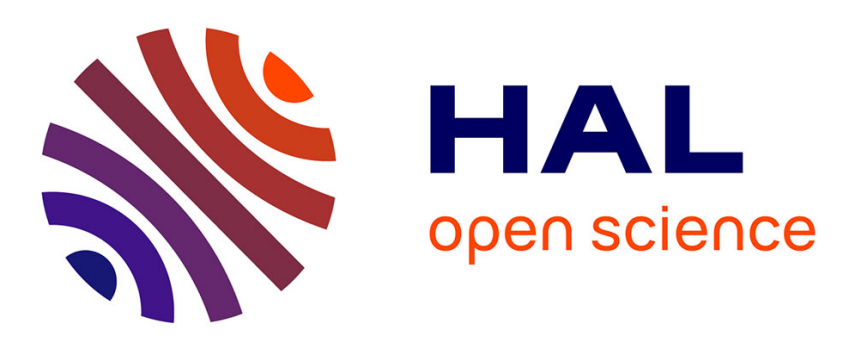

\title{
An Industrial View on LiFi Challenges and Future
}

Bastien Béchadergue, Benjamin Azoulay

\section{To cite this version:}

Bastien Béchadergue, Benjamin Azoulay. An Industrial View on LiFi Challenges and Future. 2020 12th International Symposium on Communication Systems, Networks and Digital Signal Processing (CSNDSP), Jul 2020, Porto (virtual), Portugal. 10.1109/CSNDSP49049.2020.9249584 . hal03163935

\section{HAL Id: hal-03163935 \\ https://hal.science/hal-03163935}

Submitted on 9 Mar 2021

HAL is a multi-disciplinary open access archive for the deposit and dissemination of scientific research documents, whether they are published or not. The documents may come from teaching and research institutions in France or abroad, or from public or private research centers.
L'archive ouverte pluridisciplinaire HAL, est destinée au dépôt et à la diffusion de documents scientifiques de niveau recherche, publiés ou non, émanant des établissements d'enseignement et de recherche français ou étrangers, des laboratoires publics ou privés. 


\section{An Industrial View on LiFi Challenges and Future}

\author{
Bastien Béchadergue
}

\author{
Oledcomm \\ Vélizy-Villacoublay, France \\ bastien.bechadergue@oledcomm.net
}

\author{
Benjamin Azoulay \\ Oledcomm \\ Vélizy-Villacoublay, France \\ benjamin.azoulay@oledcomm.net
}

\begin{abstract}
Light-Fidelity (LiFi) is becoming increasingly popular in both academic and industrial circles. Therefore, several companies developing LiFi products, among which Oledcomm, have emerged over the past few years so that market feedback is now available. In this paper, the recurring feedback we identified, as LiFi vendor, are first detailed. The main use cases and existing products are then reviewed, with a specific focus on the LiFiMAX solution for secure indoor networking. Finally, ongoing standardization and ecosystem development initiatives are discussed, along with some of the major technical challenges that remain.
\end{abstract}

\section{INTRODUCTION}

Global mobile traffic has been increasing rapidly for several years now. According to Cisco's annual report, the number of user equipment (UE) connected to Internet protocol (IP) networks is expected to increase from 18.4 billion in 2018 to 29.2 billion in 2023 [1]. Although this growing need for connectivity will be partially absorbed by $\mathrm{WiFi}$ and cellular networks, a massive lack of radio frequency (RF) spectrum could quickly become a major concern. At the same time, wireless networks, especially $\mathrm{WiFi}$, are increasingly subject to cybersecurity threats such as distributed denial of services (DDoS) or man-in-the middle (MITM) attacks [1].

To address these concerns, the millimeter wave spectrum is now being considered by both the WiGig and the 5G frameworks. In parallel, optical wireless communications (OWC), which use light as data carrier, have been proposed as a complementary connectivity solution. OWC can indeed rely on a spectrum that is more than 1000 times wider than the RF spectrum. It is also intrinsically secured as the light cannot pass through obstacles and the light source beams can easily be reshaped using optical systems.

In practice, the OWC field encompasses several light communication technologies. Free space optics (FSO) was the first to appear in the 1960s and now allows Gbps point-to-point terrestrial communication over several $\mathrm{km}$ using focused laser beams [2]. Short-distance infrared (IR) communications then emerged in the 1990s, especially thanks to the Infrared Data Association (IrDA), and then transformed with the emergence of white light emitting diodes (LED) into visible light communications (VLC) [3]. VLC then gave birth to optical camera communications (OCC), which consists in using a camera to receive the light data signal [4]. It also led to the concept of light-fidelity ( $\mathrm{LiFi})$ dedicated to high-speed, bidirectional, and fully networked wireless communications through light [5].

Although the term LiFi was forged in 2011, the idea of creating wireless networks through light can be traced back to at least 1979 [6] and has then seen a lot of works based on IR in the 1990s [7]. For several years now, LiFi has been attracting increasing attention from the academic world, which has been interested in topics as varied as the performance improvement with off-the-shelf or custom components [8]-[11], the study of the impact of user mobility [12], [13], but also its application to various use cases such as robotics [14].

At the same time, LiFi has also aroused the interest of the industrial world, so that several companies specializing in this field have emerged in the 2010s. Oledcomm, founded in 2012, is one of these companies and is now one of the main LiFi vendors. It has therefore acquired a certain knowledge of the LiFi market, its current limitations and future challenges, which this article aims to convey.

Section II will thus come back to the advantages of LiFi and will then describe the main vendors and use cases. In Section III, an example of a LiFi product currently on the market will be detailed. Section IV will provide an overview of the various standardization and ecosystem development initiatives. Section $\mathrm{V}$ will then suggest future directions for LiFi whereas Section VI will conclude this article.

\section{LIFI BENEFITS AND USE CASES}

\section{A. Main Benefits of LiFi}

The properties of light give undeniable advantages to LiFi. As already mentioned, it provides an extremely wide and unregulated spectrum that can be exploited to achieve very high data rates, especially by transmitting data over parallel wavelengths [8]. In parallel, LiFi provides increased security over RF technologies as light beams cannot go through obstacles and can be easily reshaped by optical systems, which reduces the risk of casual eavesdropping.

LiFi can also benefit from high spectral efficiency by pushing spatial reuse to new limits. Like other wireless communication technologies, LiFi relies on cells created by spatially distributed access points (AP) providing continuous connectivity to any compatible mobile UE. The coverage of an AP is limited by the light source properties, regulated themselves by eye safety or lighting recommendations. Consequently, LiFi cells generally have dimensions of the order of a few meters, way below the usual size of RF cells.

These reasons, added to the fact that light does not interfere with RF, make LiFi an interesting solution to complement and offload the RF spectrum, but also to provide wireless connectivity in places where RF are restricted because of electromagnetic interference or health reasons. For example, the "Loi Abeille", adopted in 2015 in France, prohibits WiFi in the areas of 
public establishments dedicated to reception, rest and activities of children under the age of three [15].

Finally, one of the latest key idea of $\mathrm{LiFi}$, is to reuse the LED lighting infrastructure already in place to provide network access, as more than $70 \%$ of the traffic actually occurs indoor [1]. This way, the growing need for connectivity could be addressed at almost no additional energy cost and without having to deploy a new and dedicated infrastructure, which would make LiFi a 'green' technology compatible with the $6 \mathrm{G}$ objectives.

\section{B. The LiFi Vendors Ecosystem}

In Europe, several organizations started to commercialize LiFi solutions in the 2010s. Among them, Oledcomm was created in 2012 as a spin out of the University Paris-Saclay, after first works on automotive VLC carried out at the Laboratoire d'Ingénierie des Systèmes de Versailles. After developing a VLC and OCC range of products, Oledcomm focused its efforts on the design of LiFi products, which is now its core activity. In 2017, the MyLiFi lamp was launched, which was primarily designed as a remotely controllable light therapy lamp that could also provide a $10 \mathrm{Mbps} \mathrm{LiFi}$ network access. Then in 2019, the LiFiMAX solution, shown on Figure 1 and detailed in Section II, was launched for indoor networking usage.

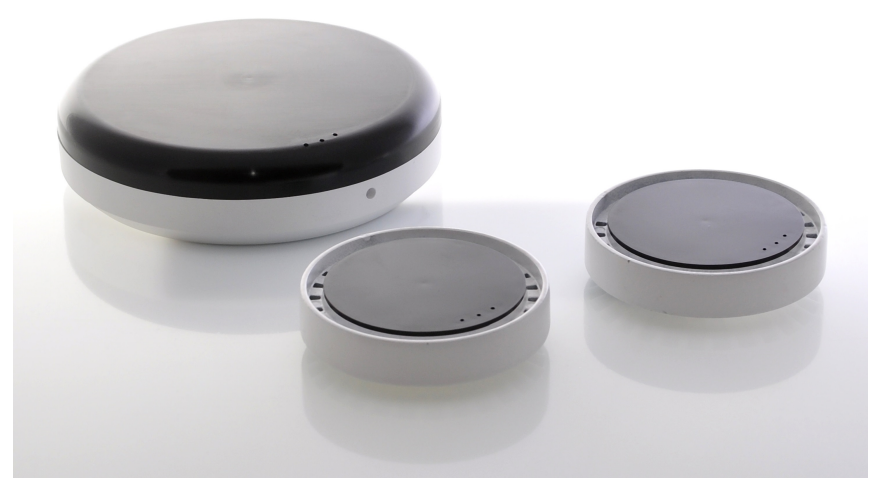

Fig. 1: The LiFiMAX AP and dongles.

Other European organizations currently propose LiFi solutions, such as the companies pureLiFi and Signify, or the Fraunhofer research institute. pureLiFi was founded in 2012, as a spin out of University of Edinburgh, and launched in 2017, together with the lighting company Lucibel, the LiFi$\mathrm{XC}$ product for indoor networking with LiFi-integrated lighting. Then in 2019, it announced the Gigabit LiFi component, a light antenna designed to be integrated directly into mobile devices. On its side, Signify was initially the lighting business unit of Royal Philips which, after some first works on VLC and OCC, acquired the start-ups Luciom and then Firefly to finally propose in 2019 its TruLiFi range of products. Finally, the Fraunhofer institute has a long research history on OWC that led independently to the Gigabit VLC module designed by the Heinrich Hertz Institute (HHI) and to the LiFi Hotspot by the Institute for Photonic Microsystems (IPMS). LiFi has also gained industrial interest outside Europe, with vendors such as VLNComm in the United States or Velmeni in India. At the same time, and as it will be further detailed in Section IV$\mathrm{E}$, several companies in the lighting and telecommunications sectors such as Nokia, Orange or Ledvance are closely following the evolution of the LiFi market even though they are not direct vendors.

\section{Market Feedback and Main LiFi Use Cases}

As one of the main LiFi vendors, Oledcomm has been able to collect interesting feedback on the actual benefits that potential users see in this technology. The first striking conclusion is that a majority of them do not attach so much importance to the lighting function, which is sometimes even seen as a constraint. Similarly, while the lack of radio spectrum is generally clearly identified as a future problem that LiFi could address, it does not seem urgent enough to justify the systematic need of this technology.

Security actually seems to be the LiFi advantage that best fits the market's expectations. While the number of cyberattacks, including DDoS or MITM attacks using unsecured WiFi networks as an entry point, grew by $776 \%$ between 2018 and 2019 [1], an increasing number of companies consider LiFi as a promising solution for secure wireless connectivity. In addition, the connection quality and reliability provided in each $\mathrm{LiFi}$ cell is greatly appreciated. Consequently, the first major use case for $\mathrm{LiFi}$ is secure wireless network access in sensitive environments such as government offices, defense institutions or strategic industries. We consider that the first entries into this market are occurring now and that it will be mature from 2023 onwards. Other office environments could then follow and reach maturity around 2025.

These needs for security and reliability have also been expressed for various other applications, which could have first market entries by 2023. A first example is industry 4.0, for which promising demonstrators have already been made, as shown in [14]. Another promising application is wireless network access for passengers in transportation. An example of such an application for aircraft cabin will be detailed in Section III-B. However, note that this list of use cases may rapidly evolve, as unexpected applications often emerge from potential users themselves.

\section{LiFIMAX, AN EXAMPLE OF LIFI SOLUTION}

\section{A. A Solution for Indoor Networking Usage}

The LiFiMAX suite is currently the main product marketed by Oledcomm. It consists of three main components: the AP, the dongle or end point (EP), and the controller. Figure 2 shows how these different elements are arranged in a typical LiFi indoor network access scenario.

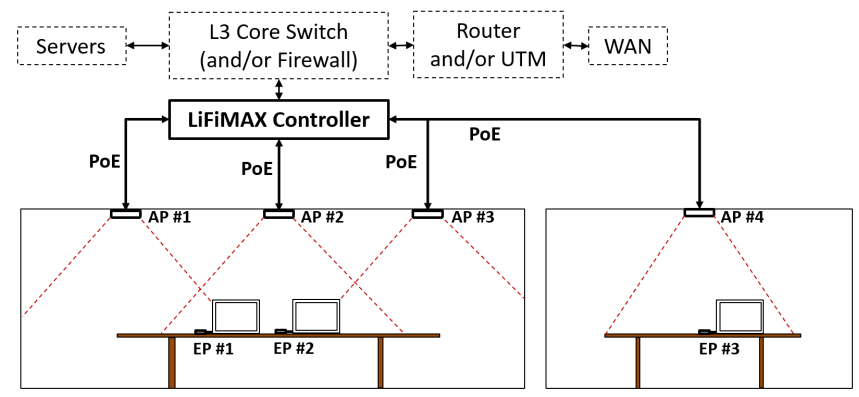

Fig. 2: The LiFiMAX solution connection diagram. 
As shown in Table I, the LiFiMAX solution is based on the G.vlc 'standard' defined by the International Telecommunication Union (ITU). Each AP can generate a cell of approximately 12 $\mathrm{m}^{2}$ (at $2 \mathrm{~m}$ height) in which a maximum of 16 devices each equipped with an EP can connect simultaneously using the time division multiple access (TDMA) protocol. One AP can then provide $100 \mathrm{Mbps}$ in both the downlink and uplink directions. Inter-cell interference zones, such as the one in which EP\#1 is located in Fig. 2, are managed. Handover from one cell to the next when the EP is mobile is therefore also supported. In addition, the wavelength of operation is in both directions 940 $\mathrm{nm}$. The LiFiMAX system is thus completely independent of any lighting source, making it easy to arrange the APs to provide complete coverage of a room. The link eventually implemented is not only secured by the light properties, but also by the possibility to add real-time advanced encryption standard (AES) between AP and EP.

TABLE I: Main characteristics of the LiFiMAX solution.

\begin{tabular}{|c|c|}
\hline Characteristic & Value \\
\hline LiFi standard & ITU G.vlc \\
\hline Wavelength of operation & $940 \mathrm{~nm}$ \\
\hline Downlink/uplink data rate & $100 \mathrm{Mbps} / 100 \mathrm{Mbps}$ \\
\hline Cell size & $12 \mathrm{~m}^{2}$ (at $2 \mathrm{~m}$ ) \\
\hline Number of users per cell & Up to 16 \\
\hline Handover & AES \\
\hline Encryption & $<7 \mathrm{~W}$ (PoE IEEE $802.3 \mathrm{af})$ \\
\hline AP nominal power (interface) & $110 \mathrm{~mm}$ diameter / 30 mm height \\
\hline AP dimensions & $<2 \mathrm{~W}$ (USB-C) \\
\hline EP nominal power (interface) & $63 \mathrm{~mm}$ diameter / 17 mm height \\
\hline EP dimensions &
\end{tabular}

The EP is plug-and-play with any device running Windows, Linux, macOS and most versions of Android or iOS, and can be connected to a single USB-A or USB-C port since its nominal power consumption is around $2 \mathrm{~W}$. The AP, on its side, consumes on average around $7 \mathrm{~W}$ and has a single IEEE 802.3af power over Ethernet ( $\mathrm{PoE})$ interface for power and data.

All the deployed APs are then interfaced with the building's network via the LiFiMAX controller. This controller can be on premise or in cluster mode and adds many features. On the one hand, it allows the management of all APs and EPs connected to the network through a dedicated user interface on which network analysis reports are displayed. The controller also supports active directory for user authentication management and the secure sockets layer/transport layer security (SSL/TLS) protocol for virtual private network (VPN) implementation. Finally, the controller enables the creation of custom virtual local area networks (VLAN).

All of these features make LiFiMAX a particularly suitable solution for setting up secure wireless networks, especially for sensitive office spaces such as those found in government, defense and corporate buildings. The architecture of this product can however be adapted to other use cases, as shown in the following section.

\section{B. Adaptation to an Aeronautical Usage}

The aeronautical use case provides an example of LiFiMAX adaptation. The aircraft environment is often considered a RF hostile environment because of the potential interference with navigation devices. In reality, WiFi is now increasingly deployed in aircraft, although it suffers from degraded performance because a cabin behaves like a Faraday cage which amplifies the multipath fading effect. LiFi is therefore considered as an alternative connectivity solution, not because it does not use RF, but because it could provide each passenger with a high-speed, low-latency, stable and secure connection.

As part of a project with Latécoère and Air France, Oledcomm has developed a LiFi solution to provide each passenger with an access to the in-flight entertainment (IFE) servers containing all the movies, music and video games available in flight. The architecture of the developed system is shown in Fig. 3(a). The IFE data stored in the 'Head End' and requested by a passenger is distributed via a 'Data Link Unit' to one of the centralized 'LiFi Modem Unit' which is thus not directly above the passenger concerned. The data is here converted from Ethernet to G.vlc by the LiFiMAX system and then distributed to the passenger via an optical fiber, itself connected to an optical system placed above the user. In the absence of UEs already $\mathrm{LiFi}$ compatible, and in order to maximize the probability of LOS, the EP is integrated directly into the seat facing the passenger and is connected to the passenger's screen. Fig. 3(b) shows on the left the optical transmit and receive interfaces connected to the optical fibers which are connected at the 'LiFi Modem Unit' level with the LED or photodiode of the LiFiMAX system. On the other hand, the right figure shows the EP integrated in the seat.

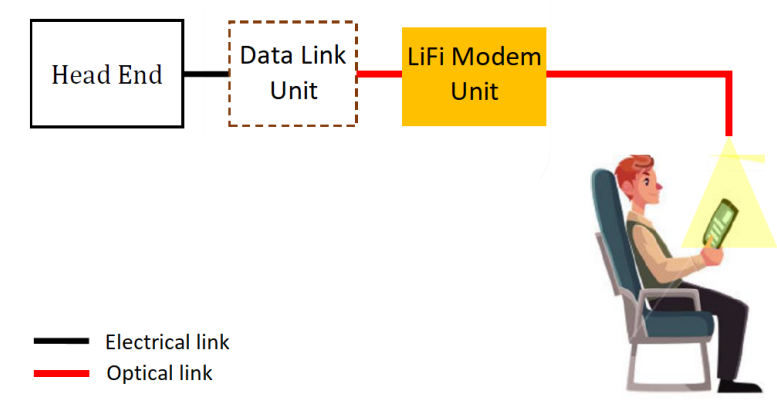

(a) Demonstrator architecture.
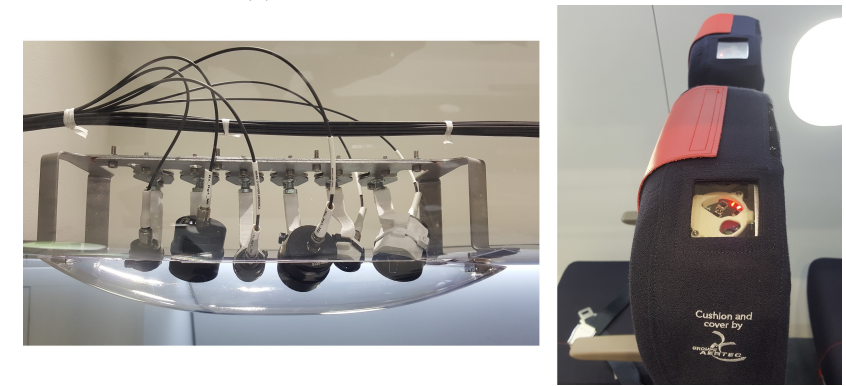

(b) LiFi remote transceiver with fiber optics backbone (left) and EP integrated into the passenger seat (right).

Fig. 3: LiFiMAX-based demonstrator for IFE network access.

This system was demonstrated for the first time during the 2019 Paris Air Show. It was then certified and installed on board an Airbus A321 operating the shuttle between Paris and Toulouse so that the first flight equipped with LiFi was performed in October 2019 and allowed 12 passengers to participate in an e-game competition. 


\section{Standardization AND ECOSYSTEM DEVElopment}

The massive deployment of a technology like LiFi depends on two fundamental cornerstones. On the one hand, standards, which are the essential prerequisite for a mass adoption of $\mathrm{LiFi}$ systems. On the other hand, LiFi vendors alone are not enough to develop the market and must therefore be integrated into a broader ecosystem including many players in various fields. Sections IV-A to IV-D review the various existing LiFi standards whereas Section IV-E gives a more detailed view of current the LiFi ecosystem.

\section{A. Early Standards}

After two first VLC standards published in 2007 by the Japan Electronics and Information Technology Industries Association (JEITA) - the JEITA CP-1221 and JEITA CP-1222 - standardization efforts were concentrated from 2009 onwards within the IEEE and more precisely within its 802.15 working group, first through the task group (TG) 7. In 2011, an IEEE 802.15.7-2011 standard inspired by ZigBee is thus published and gives a better visibility to VLC. However, this standard is little followed, so that the TG7 starts in 2015 a revision which will lead in 2018 to a new mount mainly dedicated to OCC. In the meantime, the standardization work on $\mathrm{LiFi}$ has been concentrated within the IEEE 802.15.13 group from 2017 and in parallel within the IEEE 802.11 bb group from 2018 .

\section{B. IEEE 802.15.13 for Industrial Applications}

TG13 was created as it became clear that IEEE 802.15.7 alone could not encompass OCC and LiFi. Its goal is to provide an OWC standard that supports data rates of up to 10 Gbps over $200 \mathrm{~m}$ in point-to-point and point-to-multipoint configurations for wavelength ranging from $10,000 \mathrm{~nm}$ to $190 \mathrm{~nm}$. The first key point is the definition of a main physical (PHY) layer based on DC-biased optical orthogonal frequency division multiplexing (DCO-OFDM) with adaptive bit-power-loading, derived from G.hn, and designed to optimize the link performance despite channel variations. A low power PHY layer based on pulsed modulations is also defined for the most demanding use cases in terms of consumption.

The other key point is the addition at the medium access control (MAC) level of a coordinated topology with a 'master coordinator' able to manage several 'coordinators' serving as APs to the UEs. This topology, called distributed multipleinput multiple-output (MIMO), can notably support a very fast handover, which makes it particularly suitable for very mobile applications such as industrial robots. A second, more traditional MAC is also defined to support the less restrictive mobility of enterprise applications. Table II sums up the main characteristics of the IEEE 802.15.13 standard, which should be completed by July 2020.

\section{IEEE 802.11bb for Mass-Markets}

In addition to the MAC layers just mentioned, discussions within TG13 on this subject have led to the proposal to use the 802.11 layers. It was then preferred to bring this proposal directly into 802.11, which finally led to the creation of the TGbb in May 2018. The TGbb targets LiFi links ranging from $10 \mathrm{Mbps}$ to $5 \mathrm{Gbps}$ while meeting the needs of the mass-market, and smartphones in particular. It proposes to make existing WiFi chipsets compatible with $\mathrm{LiFi}$ and thus to reuse as much as possible the 802.11 PHY and MAC layers.

Consequently, the $20 \mathrm{MHz}$ PHY layer of 802.11a has recently been defined as the common PHY mode. Discussions are also under way to define more efficient PHY layers with contributions inspired by 802.11ax on the one hand and 802.15.13 on the other hand. These different PHY modes will have to operate for wavelengths ranging from at least $800 \mathrm{~nm}$ to 1000 $\mathrm{nm}$. In parallel, the TGbb will soon start discussions on the MAC layers. The general idea is to use here again a common 802.11 MAC to ensure cross-compatibility with existing WiFi management platforms, which should allow native handover between WiFi and LiFi but also the ability to connect with the 4G/5G network management framework. All this work should be completed in 2021, as shown in Table II.

\section{ITU G.vlc}

Between 2009 and 2010, the ITU approved a first set of recommendations named G.hn for home networking, which specifies the PHY and data link layer (DLL) for operation at up 2 Gbps over telephone wiring, coaxial cables, power lines and plastic optical fiber. The PHY layer, defined in Recommendation G.9960, was actually based on DCO-OFDM and thus already suited for LiFi communication. Therefore, first LiFi demonstrations using existing G.hn chipsets were made and proved the benefits of G.9960, and especially of its bit-powerloading feature, for such applications.

In 2018, the ITU started the G.vlc project to specify the system architecture and PHY/DLL functionality of $\mathrm{LiFi}$ transceivers for high-speed indoor networking applications. This led to the publication in 2019 of a new set of PHY recommendations, called G.9991, which includes the same features as G.9960 with an additional OFDM-based PHY. On the DLL side, the same Recommendation G.9961 from G.hn is kept in G.vlc, so that G.hn chipsets are actually G.vlc-ready. It supports multiuser access for up to 16 users per domain (i.e. AP) through TDMA or frequency division multiple access (FDMA), but does not natively handle handover for the moment. However, corrigendum to ITU recommendations are regularly published with new features so that this vital function could appear and a next version of G.9961.

As highlighted by Table II, G.vlc is actually the only LiFi standard with chipsets available. This is logically reflected in the products currently on the market, since Oledcomm's LiFiMAX, Singify's TruLiFi or HHI's GigabitVLC are all based on such chipsets.

\section{E. The Light Communication Alliance}

Some LiFi vendors have already been introduced in Section II-C, along with some other key players in the LiFi sector. Most of these actors are part of the Light Communications Alliance (LCA), officially launched in December 2019 with the mission of "driving a consistent, focused and concise approach to market education that will highlight the benefits, use cases and timelines for light communications" by developing a co-operation framework involving not only LiFi vendors but also, among others, chipset providers, infrastructure companies, telecoms operators or end customers. 
TABLE II: Features comparison of the main LiFi standards.

\begin{tabular}{|c|c|c|c|}
\hline Standard & $\begin{array}{c}\text { IEEE } \\
\mathbf{8 0 2 . 1 1 b b}\end{array}$ & $\begin{array}{c}\text { IEEE } \\
\mathbf{8 0 2 . 1 5 . 1 3}\end{array}$ & ITU G.vlc \\
\hline \hline Main target & Mass-market & $\begin{array}{c}\text { Industry, } \\
\text { enterprise }\end{array}$ & $\begin{array}{c}\text { In-premise } \\
\text { network }\end{array}$ \\
\hline $\begin{array}{c}\text { Peak data } \\
\text { rate (Gbps) }\end{array}$ & 5 & 10 & 2 \\
\hline $\begin{array}{c}\text { Wavelength } \\
\text { range (nm) }\end{array}$ & $800-1000$ & $190-10,000$ & $190-5000$ \\
\hline Multiuser & Yes & Yes & Yes \\
\hline Handover & Yes & Yes & No \\
\hline $\begin{array}{c}\text { Date of } \\
\text { completion }\end{array}$ & 2021 & July 2020 & March 2019 \\
\hline Chipsets & No & No & Yes \\
\hline
\end{tabular}

The LCA is divided into three working groups (WG) with different missions. WG1 is focused on the management of the LCA and takes in charge the trademarks, incorporation, membership as well as the growth of the association and its promotion. WG2 is dedicated to marketing, that is to the promotion of OWC through conferences, white papers but also by setting-up real deployment collaborations and tracking the latest progress on the topic. Finally, WG3 takes in charge the liaison activities with the standardization bodies such as the IEEE, the ITU or the 3GPP, but also with other industrial alliances like the WiFi Alliance of the HomeGrid Forum. The final goal is to insert LiFi as a key part of the $5 \mathrm{G}$ and even more $6 \mathrm{G}$ frameworks.

This work is also crucial to define another fundamental element in the construction of the LiFi market: certification and interoperability tests. In order to move to a massive deployment of the technology, current end customers, especially industrial customers, often need a secondary supplier to rely on in case of problems with their primary supplier. Such a principle is only functional, however, if interoperability between suppliers is established. More generally, certification and interoperability are essential for any mass-market deployment, as shown by the work of the WiFi Alliance for WiFi and smartphones.

\section{Current and Future Challenges}

The previous sections show that the research conducted on $\mathrm{LiFi}$ for over 20 years is beginning to translate into business opportunities. However, the massive deployment of LiFi is still limited by many challenges of varying degrees of urgency. The list of these challenges is obviously long, so we have chosen to focus here only on those that we feel are the most crucial.

\section{A. Current Challenges}

One of the major obstacles to the expansion of $\mathrm{LiFi}$ is currently the need to connect an external device such as the LiFiMAX dongle to the UE. Such a device is indeed rather cumbersome, especially when the user is mobile. Some LiFi vendors offer smartphone or tablet shells directly integrating the $\mathrm{LiFi}$ transceiver to improve the user experience but such a solution is obviously not viable. Dedicated chipsets are thus necessary to reduce the size of the $\mathrm{LiFi}$ system but also its price and consumption. First works on LiFi chipsets have already been carried out for demonstrations [10]. However, the design and mass production of new chipsets requires significant investment, especially if digital operations such as modulation are to be integrated. It is thus necessary to carefully define the features and architecture of such chipsets, which are actually strongly dependent on the standard to be implemented.

The definition of LiFi standards is therefore the second main challenge. Standards are mainly driven by market perspective but they are based on highly technical contributions from academia and industry, which have to find the right balance between performance and features optimization for LiFi on the one hand, and integration into existing frameworks like IEEE 802.11 or ITU G.hn on the other hand. Integration into these existing frameworks is all the more crucial as it allows $\mathrm{LiFi}$ to be deployed in harmony with the RF technologies already in use. As 5G starts to be available, new contributions allowing the direct inclusion of LiFi into the 3GPP framework could thus be most beneficial.

In parallel, some functional aspects of $\mathrm{LiFi}$ have yet to be explored in more detail. More than speed, it is the availability and reliability of the connection that users appreciate in $\mathrm{LiFi}$. While these characteristics can easily be guaranteed in static use cases, they can be largely degraded by movement. The existing studies on the impact of movement [12] could therefore be extended. Furthermore, the user's movements can lead to frequent cell handover, for which several methods already exist. However, their optimization, particularly in terms of latency, remains a crucial issue [14].

\section{B. Future Challenges}

The cohabitation of $\mathrm{LiFi}$ with other RF technologies within heterogeneous networks is another major issue that will arise as $\mathrm{LiFi}$ deployment expands as part of the $6 \mathrm{G}$ framework. These heterogeneous networks raise the question of vertical handover from one technology to another, on which some work has already been done [13]. The LiFi link can indeed be cut if the photoreceiver is obstructed. On the contrary, it can be given priority over $\mathrm{WiFi}$, for example if a very secure link is required. It is thus necessary to allocate traffic between each technology according to needs and availability.

$\mathrm{LiFi}$ also aims at ensuring a high and homogeneous quality of service for all users within a cell. This quality of service is directly related to the signal-to-noise (SNR), which itself depends on the number of photons collected by the photoreceiver, so that it can degrade quickly in common use. In order to overcome this problem, a first solution is to improve the receiver sensitivity. New components such as single photon avalanche diodes (SPAD) offer interesting performances in this respect [9], but approaches such as angular diversity receivers could also be explored in greater depth, leading to programmable receivers capable of tracking the data light source [11]. An overall SNR improvement can also be achieved by optimizing this data light source. Similar to what is done in millimeter waves, the emission pattern of the source could be steered in real time in order to optimize the luminous flux transmitted to the user. While all these solutions have already been studied, they still need to be improved for use in LiFi products.

Finally, the already promising spectral efficiency of $\mathrm{LiFi}$ could be further improved. Most LiFi products use only one particular wavelength across the entire light spectrum, whereas wavelength division multiplexing (WDM) is clearly a prerequisite for achieving huge throughput. Although the solutions 
already studied seem promising [8], they still require optical filtering that is very sensitive to the angle of incidence at the receiver. The WDM implementation with simple components therefore remains a major subject of interest. The spectral efficiency could also be improved by optimizing the PHY layer and in particular the modulation, as the most commonly used scheme is currently DCO-OFDM, which sacrifices half of the subcarriers to be used with light.

\section{CONCLUSIONS}

LiFi emerged as a research field in the 2010's, in line with a long history of works on IR and visible light wireless network access. Then, it rapidly translated into the industrial sector by the creation of several specialized companies including Oledcomm, which developed a certain experience of the LiFi market. The current market feedback show that secure connection is the most appreciated advantage of LiFi. The main use case thus seems to be secure wireless network access, for which first market deployment are happening now with products like LiFiMAX.

However, the massive deployment of $\mathrm{LiFi}$ remains conditioned firstly by the progress of the standardization process. This process aims at ensuring the integration of LiFi among the other RF technologies by reducing as far as possible the various technical and market entry barriers. It will also provide a stable framework for the mass production of dedicated chipsets, which are vital for an integration of LiFi into mass-market devices such as smartphones. Another crucial process is the development of a LiFi ecosystem, started with the LCA, and including a wide variety of actors interested in LiFi for the technology promotion but also to set up its certification and interoperability processes. In parallel, many technical challenges remain to be solved in order to improve the performance of this technology and to impose it as an essential solution to the rising spectrum shortage and cybersecurity threats issues we are facing.

\section{ACKNOWLEDGMENT}

This work is supported by the European Union's Horizon 2020 research and innovation programme under the Marie Skłodowska-Curie grant agreement no 764461 (VisIoN).

\section{REFERENCES}

[1] Cisco, "Cisco Annual Internet Report (2018-2023) White Paper", 2020.

[2] M. A. Khalighi and M. Uysal, "Survey on Free Space Optical Communication: A Communication Theory Perspective," IEEE Commun. Surveys Tut., vol. 16, no. 4, pp. 2231-2258, Fourthquarter 2014.

[3] Y. Tanaka, T. Komine, S. Haruyama, and M. Nakagawa, "Indoor Visible Communication Utilizing Plural White LEDs as Lighting," in Proc. 12th IEEE PIMRC, vol. 2, Oct 2001.

[4] N. Saha, M. S. Ifthekhar, N. T. Le, and Y. M. Jang, "Survey on optical camera communications: challenges and opportunities," IET Optoelectronics, vol. 9, no. 5, pp. 172-183, Oct 2015.

[5] H. Haas, L. Yin, Y. Wang, and C. Chen, "What is LiFi?" J. Lightw. Technol., vol. 34, no. 6, pp. 1533-1544, March 2016.

[6] F. R. Gfeller and U. Bapst, "Wireless In-House Data Communication via Diffuse Infrared Radiation," Proc. IEEE, vol. 67, no. 11, pp. 1474-1486, Nov 1979

[7] J. M. Kahn and J. R. Barry, "Wireless Infrared Communications," Proc. IEEE, vol. 85, no. 2, pp. 265-298, Feb 1997.

[8] R. Bian, I. Tavakkolnia, and H. Haas, " $15.73 \mathrm{~Gb} / \mathrm{s}$ visible light communication with off-the-shelf LEDs," J. Lightw. Technol., vol.37, no.10, pp. 2418-2424, May 2019.

[9] Z. Ahmed, R. Singh, W. Ali, G. Faulkner, D. O'Brien, and S. Collins, "A SiPM-based VLC Receiver for Gigabit communication using OOK Modulation", IEEE Photonics Technol. Lett., vol. 32, no. 6, pp. 317-320, March 2020
[10] B. Fahs, A. J. Chowdhury, and M. M. Hella, "A 12-m 2.5-Gb/s Lighting Compatible Integrated Receiver for OOK Visible Light Communication Links", J. Light. Technol., vol. 34, no 16, pp. 3768-3775, Aug 2016.

[11] I. Takai, T. Harada, M. Andoh, K. Yasutomi, K. Kagawa, and S. Kawahito, "Optical Vehicle-to-Vehicle Communication System Using LED Transmitter and Camera Receiver", IEEE Photonics J., vol. 6, no 5, pp. 1-14, Oct 2014.

[12] M. D. Soltani, A. A. Purwita, Z. Zeng, H. Haas, and M. Safari, "Modeling the random orientation of mobile devices: Measurement, analysis and lifi use case," IEEE Trans. Commun., vol. 67, no. 3, pp. 2157-2172, 2019.

[13] X. Wu, and H. Haas, "Load Balancing for Hybrid LiFi and WiFi Network: To Tackle User Mobility and Light-Path Blockage", IEEE Trans. Commun., vol. 68, no. 3, pp. 1675-1683, March 2020.

[14] P. W. Berenguer, P. Hellwig, D. Schulz, J. Hilt, G. Kleinpeter, J. K. Fischer, and V. Jungnickel, "Real-Time Optical Wireless Communication: FieldTrial in an Industrial Production Environment," in Proc. 2018 ECOC, Sept 2018.

[15] "LOI n 2015-136 du 9 février 2015 ("Loi Abeille")", JORF, no. 0034, p. 2346, Feb 2015. 